\title{
Erratum: Induced magnetic two-dimensionality by hole doping in the superconducting infinite-layer nickelate $\mathrm{Nd}_{1-x} \mathrm{Sr}_{x} \mathrm{NiO}_{2}$ [Phys. Rev. B 101, 064513 (2020)]
}

\author{
Siheon Ryee, Hongkee Yoon, Taek Jung Kim, Min Yong Jeong, and Myung Joon Han (1)
}

(Received 3 January 2022; published 9 February 2022)

DOI: 10.1103/PhysRevB.105.059902

While the conclusion of this paper is unaffected, two errors exist in Figs. 1(b) and 3. First, in Fig. 1(b), the density of states (DOS) of O-2 $p$ characters is two times smaller than the correct values. The corrected version of this figure is Fig. E1.

Second, we have found that the values of $J_{i j}$ presented in Fig. 3 are half of the correct ones. The corrected version of this figure is Fig. E2. We have also realized that the definition of magnetic exchange coupling $J_{i j}$ is omitted in this paper, which can cause confusion to readers. Our $J_{i j}$ is defined as the coupling strength of the following spin Hamiltonian,

$$
H=-\sum_{i \neq j} J_{i j} \mathbf{e}_{i} \cdot \mathbf{e}_{j},
$$

where $\mathbf{e}_{i(j)}$ denotes a unit vector on a site $i(j)$. Thus, the magnitude of the spin moment of $\mathrm{Ni}$ is absorbed into $J_{i j}$. Accordingly, the values of $J_{i j}$ presented in Fig. E2 should be interpreted with this caveat in mind.

It is worth noting that one can also follow a different (but more commonly adopted) form of the spin Hamiltonian which reads

$$
H=\frac{1}{2} \sum_{i \neq j} \widetilde{J}_{i j} \mathbf{S}_{i} \cdot \mathbf{S}_{j},
$$

where $\mathbf{S}_{i(j)}$ denotes a spin moment on a site $i(j)$. Thus, taking the magnitude of spin moments to be $\left|\mathbf{S}_{i(j)}\right|=1 / 2$, which may be a relevant value for infinite-layer nickelates, one gets the following relation,

$$
\widetilde{J}_{i j}=-8 J_{i j}
$$

Hence, in this convention, $\widetilde{J}_{i j}$ between in-plane nearest neighbors of updoped $\mathrm{NdNiO}_{2}$, namely $\widetilde{J}_{1}^{\mathrm{n}}$, becomes $\widetilde{J}_{1}^{\mathrm{n}}=-8 J_{1}^{\text {in }}=$ $50.24 \mathrm{meV}$ because $J_{1}^{\text {in }}=-6.28 \mathrm{meV}$. See Table E1 for first-neighbor in-plane and out-of-plane couplings.

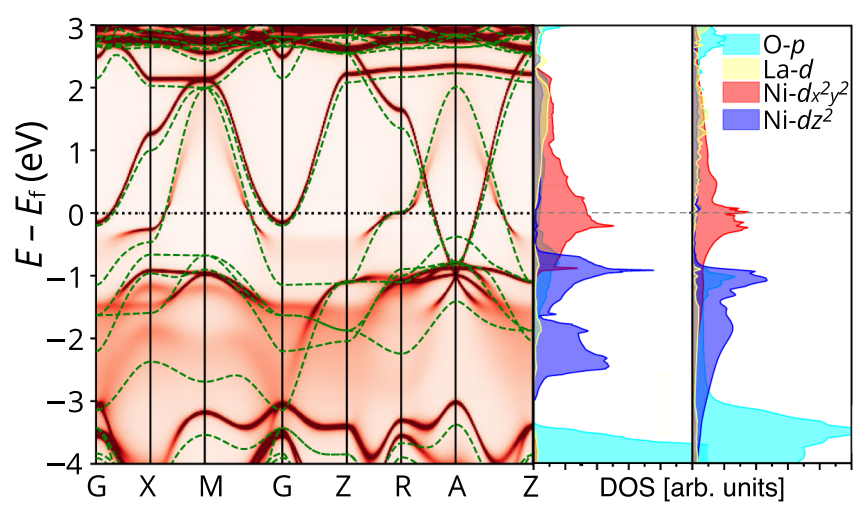

FIG. E1. In the leftmost panel, the calculated band dispersion by LDA and LDA+DMFT is presented, respectively, with dashed green lines and color intensity. Dark and bright red regions represent the larger and smaller spectral weights, respectively, obtained by LDA+DMFT. The right two panels show the calculated DOS by LDA (middle) and by LDA+DMFT (rightmost). 
(a)

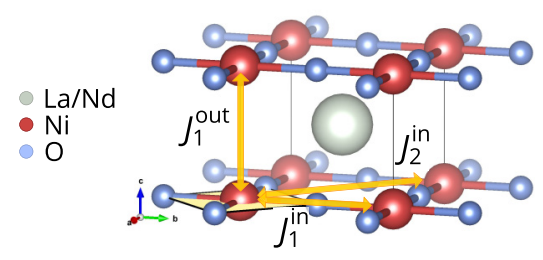

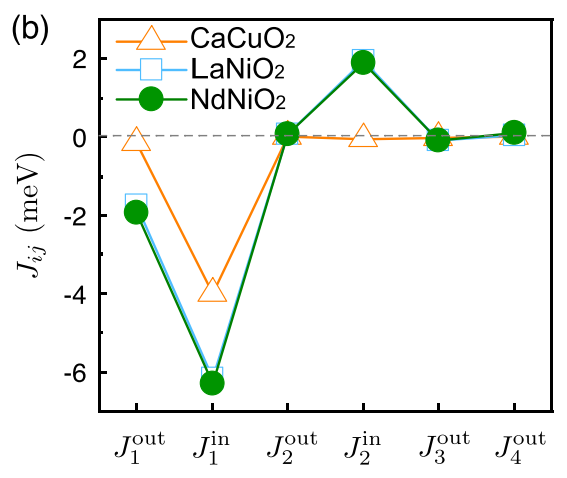

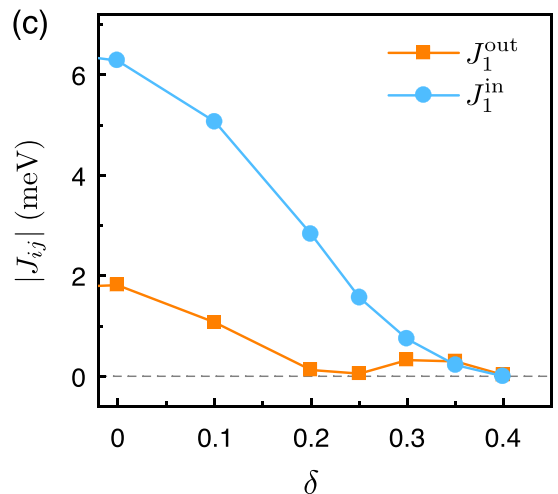

FIG. E2. (a) Crystal structure of $R \mathrm{NiO}_{2}(R=\mathrm{La}, \mathrm{Nd})$. The yellow arrows depict the first-neighbor out-of-plane $\left(J_{1}^{\text {out }}\right)$, the first-neighbor in-plane $\left(J_{1}^{\text {in }}\right)$, and the second-neighbor in-plane $\left(J_{2}^{\text {in }}\right)$ magnetic exchange couplings. (b) The calculated $J_{i j}$ profile as a function of Ni-Ni (or $\mathrm{Cu}-\mathrm{Cu}$ ) interatomic distance for $\mathrm{LaNiO}_{2}$ (blue open squares), $\mathrm{NdNiO}_{2}$ (green solid circles; with $U=8 \mathrm{eV}$ for $\mathrm{Nd}-4 f$ ), and CaCuO 2 (open triangle; orange). The negative and positive signs of $J_{i j}$ represent the AFM and ferromagnetic couplings, respectively. We used GGA-PBE for $\mathrm{LaNiO}_{2}$ and $\mathrm{NdNiO}_{2}$ and charge-only-density version of GGA $+U(U=8$ and $J=0.8 \mathrm{eV}$ with $\mathrm{AMF}$ double-counting) for CaCuO . (c) The calculated $J_{1}^{\text {out }}$ and $J_{1}^{\text {in }}$ as a function of hole doping $\delta$. For comparison, their absolute values are presented.

TABLE EI. The calculated magnetic exchange coupling constants of undoped $\mathrm{NdNiO}_{2}(\delta=0)$ obtained from GGA-PBE for two different definitions of the spin Hamiltonian.

\begin{tabular}{lrr}
\hline \hline & $J_{i j}[\mathrm{Eq} .(\mathrm{E} 1)]$ & $\widetilde{J}_{i j}[\mathrm{Eq} .(\mathrm{E} 2)]$ \\
\hline First-neighbor in-plane & $-6.28 \mathrm{meV}$ & $50.24 \mathrm{meV}$ \\
First-neighbor out-of-plane & $-1.92 \mathrm{meV}$ & $15.37 \mathrm{meV}$ \\
\hline \hline
\end{tabular}

